\title{
A Cancellation-Free Symbolic Sensitivity Technique Based on Network Determinant Expansion
}

\author{
Vladimir Filaretov, Konstantin Gorshkov, and Sergey Kurganov \\ Department of Electrical Engineering, Ulyanovsk State Technical University, 32 Severny Venets Street, Ulyanovsk 432027, Russia \\ Correspondence should be addressed to Vladimir Filaretov; vvfil@mail.ru, Konstantin Gorshkov; k.gorshkov@ulstu.ru \\ and Sergey Kurganov; sakurganov@mail.ru
}

Received 28 July 2014; Revised 30 November 2014; Accepted 30 November 2014

Academic Editor: Ramón M. Rodríguez-Dagnino

Copyright (C) 2015 Vladimir Filaretov et al. This is an open access article distributed under the Creative Commons Attribution License, which permits unrestricted use, distribution, and reproduction in any medium, provided the original work is properly cited.

\begin{abstract}
The generalization of Bode's sensitivity analysis technique for all types of the transfer functions and circuit elements is presented in the paper. The proposed formulae for first- and second-order symbolic sensitivity calculation provide the compact size of obtained expression and have the advantages of cancellation-free sum-of-product terms and matrix-free computation. This is achieved by means of the concept of high order summative cofactors and the generalized parameter extraction method. The proposed technique is implemented in symbolic circuit analysis program Cirsym. Illustrative example on symbolic sensitivity circuit analysis and comparison of the presented technique with the transimpedance method and the method based on the modified Coates flow-graph are given.
\end{abstract}

\section{Introduction}

The influence of network components on transfer function is expressed by sensitivities [1]. Sensitivity analysis is an important part of analog circuit design process. There are many methods of numerical calculation of circuit sensitivities, but only symbolic analysis of circuit sensitivity provides the way to get analytical sensitivity function of all interested circuit parameters. This is certainly advantageous in such applications as circuit parameters optimization and Monte Carlo simulation.

First researches on symbolic approach to sensitivity analysis of analog circuit were presented in [1-5], but those heuristic algorithms were not efficient enough and had limitations by the circuits scale.

Nowadays several more effective symbolic sensitivity analysis techniques have been developed. Some of them are based on sequence of expression computation such as twoport transimpedance method [6]. But the generated expressions are not compact enough, do not fully exploit sharing of subexpressions, cannot be easily manipulated, and complicate the circuit insight.

The others methods are based on implementation of graph theory for symbolic circuit analysis [7-10]. In particular, the work in [7] shows the technique based on the modified Coates flow-graph for performing sensitivity analysis. However, the proposed concept requires the nodal admittance matrix expansion, which may consist of the equal summands with opposite sign. It leads to appearing of the cancellation sum-of-product terms. In addition, the usage of graph-based methods does not provide the optimal size of obtaining expression.

Another widespread approach is implementation of the modified nodal matrix $[10,11]$. But obtained results are not compact and include cancellations so the expressions often present only in semisymbolic form.

In this paper we propose a cancellation-free symbolic sensitivity technique for computation of compact expression. The first- and second-order symbolic sensitivities formulas for all types of the transfer functions and circuit elements are derived by means of the concept of higher order summative cofactors (HOSC) [12-15] and generalized parameter extraction method (GPEM) [16-21] to avoid drawbacks of the previous methods mentioned above.

The rest of this paper is organized as follows. In Section 2 we explain the basic idea of sensitivity analysis by means of GPEM and derive the formulae for symbolic sensitivity of 


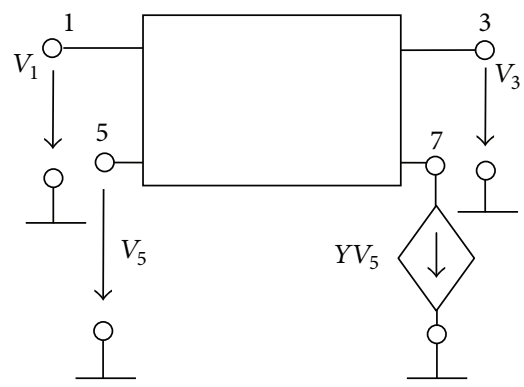

FIgURe 1: Test circuit.

different transfer functions with respect to controlled sources (CS) and two ports. The process of obtaining symbolic expressions can be easily automated. The example of the AC sensitivity analysis is presented in Section 3. In Section 4 the comparison of the presented method with the transimpedance method and the method based on the modified Coates flow-graph is performed. Section 5 concludes this paper.

\section{Theoretical Basis of the Proposed Method}

2.1. Symbolic Approach for Sensitivity Analysis. The sensitivity computation of the transfer function of $T(s)$ with respect to change of the parameter $X$ requires finding the corresponding derivative:

$$
S(H(s), X)=\frac{d H(s)}{H(s)} \frac{X}{d X} .
$$

The study of network response by means of formula (1) is acceptable only when changes in its parameter $X$ are small. When some element's parameters change significantly, this approximated result may be unsatisfied. Analytical sensitivity analysis would be much easier by using a symbolic approach than by a numerical approach where computation cost is a key concern.

If we consider the transfer function as $T(s)=N(s) / D(s)$ formula (1) will transform into expression proposed by Hoang [5]:

$$
S(T(s), X)=X\left(\frac{d N(s)}{N(s)}-\frac{d N(s)}{D(s)}\right) .
$$

Calculation of the transfer function derivatives leads to appearance of multiple algebraic cofactors with different signs and thus they may contain many cancellations. The well-known technique proposed by Bode [1] presented the sensitivity function $T(s)$ in form of ratio of determinant and cofactors of nodal admittance or loop impedance matrix multiplication. For example, the sensitivity of $T(s)=V_{3}(s) / V_{1}(s)$ of the circuit in Figure 1 is expressed as follows:

$$
S_{X}^{T}=-X \frac{\Delta_{15} \Delta_{73}}{\Delta \Delta_{13}}
$$

where $X$ is a transfer impedance $Z$ of current controlled voltage source (CCVS) or transfer admittance of voltage controlled current source (VCCS), $\Delta$ is a determinant of loop impedance matrix or $\Delta=\Delta_{11}$ for nodal admittance matrix, and $\Delta_{11}, \Delta_{15}, \Delta_{13}$, and $\Delta_{73}$ are cofactors.

Expression (3) derived from (1) by means of Jacobi identity in form of

$$
\Delta_{13,75} \Delta=\Delta_{13} \Delta_{75}-\Delta_{15} \Delta_{73},
$$

where $\Delta_{13,75}$ is a second-order cofactor. The rows and columns with numbers 1 and 3, 5, and 7 correspond to input and output variables (controlled and controlling variables of controlled source).

Sensitivity analysis by means of Bode's technique leads to appearance of cancellations because of multiple entries of elements parameters into the matrix. The sensitivity computation formulae with numbered ports of multiport circuit model presented in [5] are devoid of this drawback. But the tedious combinatorial search of the transfer function from one port to another is needed to use them.

We propose in this paper the generalization of Hoang formula and Bode's technique for all types of transfer functions and circuit elements, based on implementation of the concept of higher order summative cofactors (HOSC) [12-15] instead of ordinary algebraic cofactors and computation of HOSC by means of GPEM [16-21].

\subsection{Higher Order Summative Cofactors and Generalized} Parameter Extraction Method. The higher order cofactor is a cofactor of a cofactor. The $n$th order cofactor is represented by a symbol $\Delta_{r_{1}, k_{1}, r_{2}, k_{2}, \ldots, r_{n}, k_{n}}$, where $r_{1}, r_{2}, \ldots, r_{n}$ and $k_{1}, k_{2}, \ldots, k_{n}$ are the numbers of deleted rows and columns, respectively. The summative cofactors are cofactors with at least one deletion in a form $(a+d)$ or $(a-d)$, which means the following: add or subtract the row (column) $a$ to or from the row (column) $d$. For example the summative cofactors of the same matrix can be expressed as follows [15]:

$$
\Delta_{(a+b)(c+d)}=\Delta_{a c}-\Delta_{a d}-\Delta_{b c}+\Delta_{b d},
$$

where $\Delta_{a c}=\Delta_{(a+0)(c+0)}, \Delta_{a d}=\Delta_{(a+0)(d+0)}$, and so on.

In accordance with GPEM basis [16-21] we consider the sums of row (column) indexes as a result of nullator (norator) connection to the network nodes corresponding to the indexes.

Let us express the voltage gain function for circuit model in Figure 2(a) as follows:

$$
T(s)=\frac{V_{\text {out }}(s)}{V_{\text {in }}(s)}=\frac{\Delta_{(1+2)(3+4)}}{\Delta_{(1+2)(1+2)}},
$$

where HOSC in the numerator is the determinant of the network, in which the independent source and arbitrary response are replaced by oriented nullor [16] and HOSC in the denominator is the determinant of the network, in which the independent excitation and the arbitrary response are zero.

The calculation procedure of the network determinants is based on the recursive usage of the parameter extraction formula [16]:

$$
\Delta=\chi \Delta(\chi \longrightarrow \infty)+\Delta(\chi=0)
$$




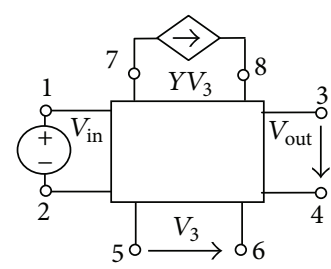

(a)

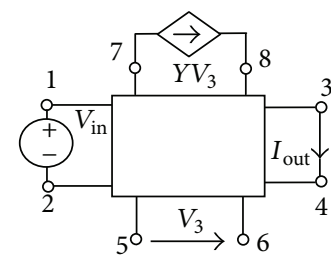

(b)

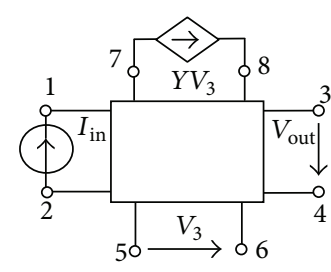

(c)

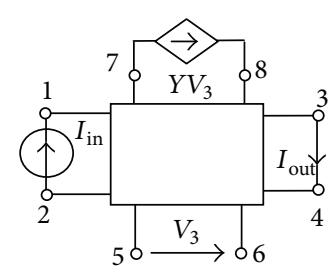

(d)

Figure 2: The circuit for sensitivity computation of transfer functions: voltage gain (a), transfer admittance (b), transfer impedance (c), and current gain (d).

where $\chi$ is a parameter of arbitrary linear circuit element and $\Delta(\chi \rightarrow \infty)$ and $\Delta(\chi=0)$ correspond to the determinants of the circuit matrix in which the parameter of extracted elements $\chi \rightarrow \infty$ or $\chi=0$, respectively.

The parameter extraction formula (7) is applicable for determinant calculation of the network consisting of any kind of linear active circuit models including the pathological elements (nullors and pathological mirrors). In case of nullor extraction the special formula was proposed in [16]

$$
\Delta= \pm \Delta_{n}
$$

where $\chi$ is the determinant of the circuit matrix after the extraction procedure of the nullor number $n$. Equation (8) means that the nullor extraction will change the sign of the initial determinant. The choice of the sign depends on the orientation of the nullator and norator. If these elements have got the same orientation with respect to the basic node that the sign will be positive, in the opposite case the sign will be negative.

The procedure of nullor extraction can be formalized by the following steps.

(1) The choice of the supporting nodes: first of the supporting nodes should be connected to norator and the second to nullator. If there is a common node of a nullator and a norator it must be chosen as single supporting node. Note that supporting node may correspond to the ground node.

(2) The terminals of non-extracted norators and current sources connected to supporting node are moved to the opposite node of extracted norator. In that case the non-extracted nullators and controlling voltages keep connections to supporting node. Then in the same way, the terminals of non-extracted nullators and controlling voltages connected to supported node are moved to the opposite node of extracted nullator. In that case non-extracted norators and current sources keep connections to supporting node.

(3) A norator and a nullator of the extracted nullor are deleted from circuit. In case of two supporting nodes they must combine.

(4) If extracted norator and nullator have got the same orientation with respect to the supporting node then the determinant sign will be positive. Otherwise the sign will be negative. In case of two supporting nodes the inverted rule is needed.
Formula (8) can be implemented not only for the nullor extraction from circuit model, but also for Coates flow-graph modification as shown in Section 4.

The generalized parameter extraction method does not have any limitation by circuit size. The GPEM has been realized by Filaretov in the program Cirsym (circuit simulator) as a part of the software tool SCAD. The program can be downloaded from http://intersyn.narod.ru/. The symbolic expressions obtained by means of Cirsym are comparable by the number of arithmetical operations with computation results by means of the professional mathematic programs as Maple and Matlab [22].

As can be seen from the comparison of symbolic expressions of notch filter transfer function [23] presented in "Symbolic circuit analysis: Library of benchmark circuits" (http://rodanski.net/ben/work/symbolic/index.htm) the result of program Cirsym named by Filaretov 2 is second ranked. Also this result was replicated in [24] as example of compact size expression.

2.3. Derivation of Symbolic Sensitivity Computation Formulae. Let us express the transfer function (6) by means of parameter extraction formula as

$$
T(s)=\frac{Y \Delta_{(1+2)(3+4),(7+8)(5+6)}+\Delta_{(1+2)(3+4)}^{0}}{Y \Delta_{(1+2)(1+2),(7+8)(5+6)}+\Delta_{(1+2)(1+2)}^{0}},
$$

and then expressed the Hoang formula (2) as follows:

$$
S_{Y}^{T}=Y \cdot\left(\frac{\Delta_{(1+2)(1+2)}^{\infty}}{\Delta_{(1+2)(1+2)}}-\frac{\Delta^{\infty}}{\Delta}\right) .
$$

Note, that upper indexes $\langle\langle 0\rangle\rangle$ and $\langle\langle\infty\rangle\rangle$ in formulas (9) and (10) mean that those HOSC are derived with $Y=0$ and $Y \rightarrow \infty$ correspondingly. In case of controlled source the operation $\chi \rightarrow \infty$ leads to substitution of CS by the nullor.

Formula (10) is applicable for sensitivity computation of the arbitrary circuit elements. Just replace the $\langle\langle Y\rangle\rangle$ by $\langle\langle\chi\rangle\rangle$.

Now let us consider the transformation of Bode formula (1) by substitution of expression (9) and cancellation by $\Delta_{(1+2)(1+2)}$ :

$$
\begin{aligned}
S_{Y}^{T}= & Y \frac{\Delta_{(1+2)(3+4),(7+8)(5+6)} \Delta_{(1+2)(1+2)}}{\Delta_{(1+2)(1+2)} \Delta_{(1+2)(3+4)}} \\
& -Y \frac{\Delta_{(1+2)(3+4)} \Delta_{(1+2)(1+2),(7+8)(5+6)}}{\Delta_{(1+2)(1+2)} \Delta_{(1+2)(3+4)}} .
\end{aligned}
$$


TABLE 1: Sensitivity symbolic calculation formulae.

\begin{tabular}{lcc}
\hline Number & Sensitivity type & Formula \\
\hline 1 & $S_{\chi}^{T}$ & $-\frac{\chi \Delta_{(1+2)(5+6)} \Delta_{(1+2)(1+2),(7+8)(3+4)}}{\Delta_{(1+2)(1+2)} \Delta_{(1+2)(3+4)}}$ \\
2 & $S_{\chi}^{Y}$ & $-\frac{\chi \Delta_{(1+2)(5+6)} \Delta_{(7+8)(3+4)}}{\Delta \Delta_{(1+2)(3+4)}}$ \\
\hline 3 & $S_{\chi}^{B}$ & $S_{\chi}^{Z}$ \\
\hline
\end{tabular}

We obtain the transfer function sensitivity expression with respect to a VCCS parameter. It is possible to minimize the numerator by means of Jacobi identity. We need to use the $\operatorname{HOSC} \Delta_{(1+2)(1+2)}$ instead of determinant $\Delta$ :

$$
S_{Y}^{T}=-Y \frac{\Delta_{(1+2)(5+6)} \Delta_{(1+2)(1+2),(7+8)(3+4)}}{\Delta_{(1+2)(1+2)} \Delta_{(1+2)(3+4)}} .
$$

As can be seen, $\Delta_{(1+2)(1+2)}$ is a numerator of transfer function from circuit input to controlling voltage of VCCS, $\Delta_{(1+2)(1+2),(7+8)(3+4)}$ is a numerator of transfer function from controlled current source to circuit output, and $\Delta_{(1+2)(1+2)} \Delta_{(7+8)(3+4)}$ is a product of numerator and denominator of voltage gain.

The process of sensitivity computation with respect to other elements and/or for different transfer function types is the same. In Table 1 the generalized sensitivity formulae are proposed.

Note that the formula in first line of Table 1 is appropriate not only for voltage gain, but also for transfer impedance function. Let us show it for circuit in Figure 2(b):

$$
Y(s)=\frac{I_{\text {out }}(s)}{V_{\text {in }}(s)}=\frac{\Delta_{(1+2)(3+4)}}{\Delta_{(1+2)(1+2)}} .
$$

Even if output nodes of transfer impedance function in Figure 2(b) are connected in contrast to Figure 2(a), it is obvious that expression (13) is equal to (6). The formulae for transfer impedance (Figure 2(c)) and current gain (Figure 2(d)) are similar like expressions (6) and (13):

$$
\begin{aligned}
& Z=\frac{V_{\text {out }}}{I_{\text {in }}}=\frac{\Delta_{(1+2)(3+4)}}{\Delta}, \\
& B=\frac{I_{\text {out }}}{I_{\text {in }}}=\frac{\Delta_{(1+2)(3+4)}}{\Delta} .
\end{aligned}
$$

The denominator of any sensitivity formulae in Table 1 is a product of initial transfer function numerator and denominator and the numerator of the sensitivity formulae is a product of numerators of transfer functions from circuit input to controlled branch and from controlling source to circuit output.

The proposed formulae are easy to use for sensitivity computation of two ports. For example, if we change indexes 7 and 8 to 5 and 6 correspondingly, we will obtain the sensitivity function for admittance element:

$$
S_{Y}^{T}=-Y \frac{\Delta_{(1+2)(5+6)} \Delta_{(1+2)(1+2),(5+6)(3+4)}}{\Delta_{(1+2)(1+2)} \Delta_{(1+2)(3+4)}} .
$$

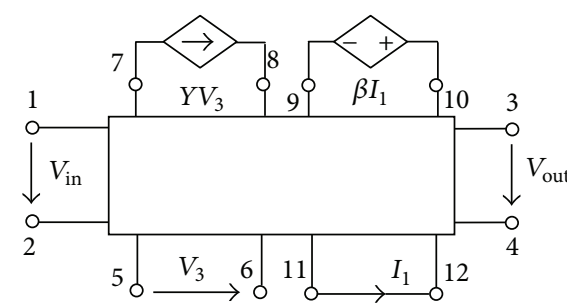

FIgURE 3: The test circuit for second-order transfer impedance sensitivity computation.

The formula for transfer function sensitivity with respect to impedance parameter $(\chi=Z)$ can be derived in the same way. We just have to replace the number of index 8 by 6 .

For sensitivity of the input impedance or input admittance functions derivation it is necessary to replace indexes 3 and 4 by 1 and 2 correspondingly.

\subsection{The Special Topological Cases of Sensitivity Formulae} Simplification. If the voltage source of CS is connected in parallel with a voltage output port or if the current source of CS is connected in series with a current output port, we can simplify the sensitivity formulae like that shown in Table 2 . As it can be seen, the HOSC indexes 3 and 4 correspond to 7 and 8. Taking into account the identity $\chi \Delta_{(1+2)(5+6)}=\Delta_{(1+2)(7+8)}$ we obtain the expressions consisting of just two HOSC.

In the same way, we can easily simplify the sensitivity formulae for other special cases if the input excitation transfers directly to the controlling branch of CS, if the controlled voltage source is connected to the input voltage port in series or the controlled current source is connected in parallel with input current source, and if the controlling voltage of CS has the same port as output circuit voltage or the controlling current of CS corresponds to output current port.

2.5. The Second-Order Sensitivity Computation. Let us consider the process of computation of second-order sensitivity function. The repeated differentiation of first-order formulae from Table 1 is needed in this case. So the second-order transfer impedance sensitivity of circuit in Figure 3 with respect to the VCCS and CCVS parameters can be expressed as follows:

$$
\begin{aligned}
S_{\beta Y}^{Z}=(\beta Y & {\left[\Delta_{(1+2)(5+6)} \Delta_{(7+8)(9+10),(1+2)(1+2)}\right.} \\
& \times \Delta_{(11+12)(3+4),(1+2)(1+2)}+\Delta_{(1+2)(9+10)} \\
& \left.\left.\times \Delta_{(11+12)(5+6),(1+2)(1+2)} \Delta_{(7+8)(3+4),(1+2)(1+2)}\right]\right) \\
& \times\left(\Delta^{2} \Delta_{(1+2)(3+4)}\right)^{-1} .
\end{aligned}
$$

We can see the product of squared determinant and the transfer function numerator in formula denominator and two terms in numerator. The first term here consists of the numerators of transfer functions, from circuit input to controlling voltage of VCVS, from voltage source of VCVS 
TABLE 2: The simplified sensitivity formulae in case of controlled source connecting with circuit output.

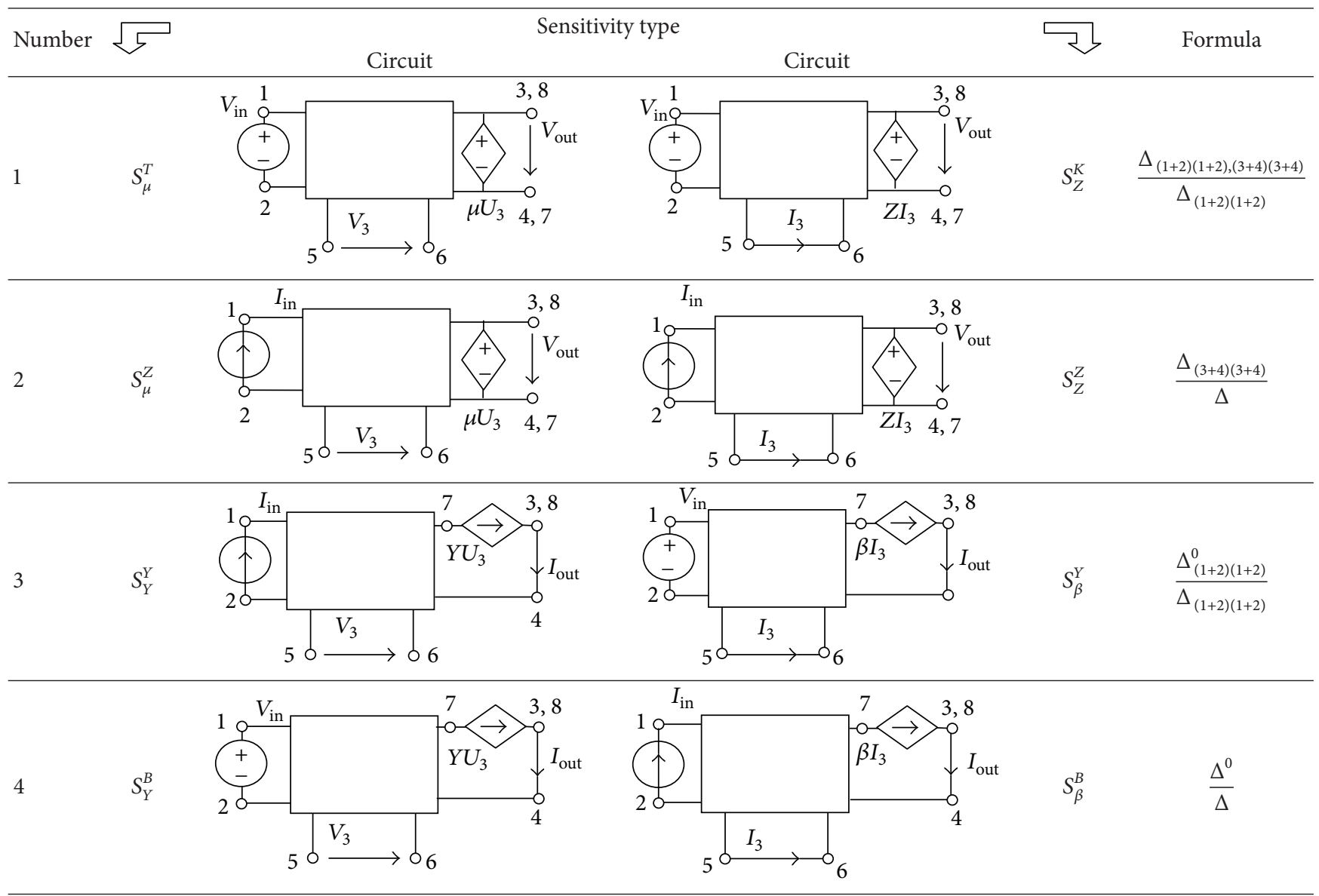

to controlling current of CCCS, and from current source of CCCS to circuit output. For the second term we take into account that succession of excitation transfer from input to output of circuit is different. In that case the signal transfers firstly to CCCS and then to VCVS.

For the purpose of obtaining the second-order sensitivity function in Hoang form we just need to differentiate and represent by means of HOSC formula (2). So for the circuit presented in Figure 3 the sensitivity expression for voltage transfer function with respect to parameters of CS is obtained as follows:

$$
\begin{aligned}
S_{\beta Y}^{T}= & \left(\beta Y \left[\Delta_{(1+2)(3+4)}^{\infty} \Delta^{2}-\Delta^{Y \rightarrow \infty} \Delta_{(1+2)(3+4)}^{\beta \rightarrow \infty} \Delta\right.\right. \\
& -\Delta_{(1+2)(3+4)}^{Y \rightarrow \infty} \Delta^{\beta \rightarrow \infty} \Delta-\Delta_{(1+2)(3+4)} \Delta^{\infty} \Delta \\
& \left.\left.+2 \Delta_{(1+2)(3+4)} \Delta^{\beta \rightarrow \infty} \Delta^{Y \rightarrow \infty}\right]\right) \\
& \times\left(\Delta^{2} \Delta_{(1+2)(3+4)}\right)^{-1}
\end{aligned}
$$

where upper indexes $\langle\langle Y \rightarrow \infty\rangle\rangle$ and $\langle\langle\beta \rightarrow \infty\rangle\rangle$ mean that those HOSC are derived with $Y \rightarrow \infty$ and $\beta \rightarrow \infty$ correspondingly. The index $\langle\langle\infty\rangle\rangle$ means that this HOSC is derived with both parameters tending to infinity.

\section{Symbolic Sensitivity Analysis Examples}

Let us consider the voltage transfer function symbolic AC sensitivity analysis of second-order low-pass filter shown in Figure 4 [25]. The values of parameters elements are as follows: $C_{1}=18,7571 \mathrm{nF} ; C_{2}=9,37857 \mathrm{nF} ; G_{1}=G_{2}=1 \mathrm{mS}$; $\mu=1$.

As can be seen, the circuit topology makes it possible to simplify sensitivity computation with respect to VCVS parameter by means of formula from the first line of Table 2. Taking into account the nodes numeration of filter circuit in Figure 4 the sensitivity function can be expressed as follows:

$$
S_{\mu}^{T}=\frac{\Delta_{(1+0)(1+0),(2+0)(2+0)}}{\Delta_{(1+0)(1+0)}}=\frac{\Delta_{11,22}}{\Delta_{11}} .
$$

The HOSC expansion is performed by means of GPEM. In accordance with (7) the VCVS parameter is extracted from circuit as shown in Figure 7.

The second summand of expression (Figure 7) is correspond to the HOSC $\Delta_{11,22}$, the numerator of formula (18). It is desirable to obtain the polynomial symbolic expression so the circuit expansion is performed by extracting capacitors admittances.

Note, that admittances extraction leads to appearance of two subcircuits with short circuit and deleting of the element correspondingly. The determinant of the first circuit 


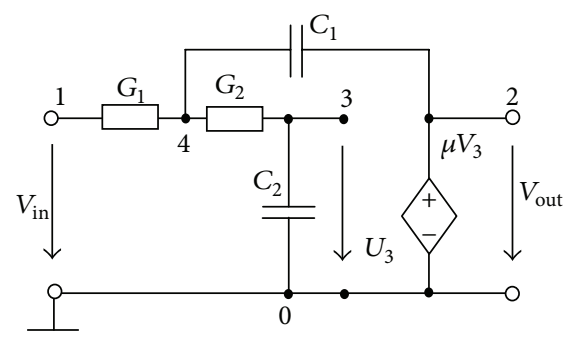

FIGURE 4: Second-order low-pass filter.

in (Figure 8) is equal to unity because both admittances connected in short circuit. After expansion of element $G_{2}$ from second circuit in (Figure 8) we obtain the determinant of short-circuited admittance $G_{1}$ in the same way. In the third circuit with parallel admittances the determinant is equal to the elements sum. And the last circuit determinant is a product of $G_{2}$ and $G_{1}$.

The symbolic determinant expression for the first summand in (Figure 7) is shown in Figure 9

For computation of expression (Figure 9) the following procedures were done: deletion of capacities admittance $s C_{2}$ connecting in parallel with nullator, parameter extraction of admittance $s C_{1}$ connecting in series with nullator, followed by deletion of the admittance $G_{1}$ connecting in parallel with nullator. As a result we obtain norator-nullator pair connected in opposite direction and determinant of that kind of circuit is equal to " -1 ."

Now we need to substitute the symbolic expression from (Figures 8 and 9) in (18) to obtain the voltage gain sensitivity function:

$$
S_{\mu}^{T}=\frac{s^{2} C_{1} C_{2}+s\left[C_{1} G_{2}+C_{2}\left(G_{1}+G_{2}\right)\right]+G_{1} G_{2}}{s^{2} C_{1} C_{2}+s\left[(1-\mu) C_{1} G_{2}+C_{2}\left(G_{1}+G_{2}\right)\right]+G_{1} G_{2}} .
$$

The derivation process of sensitivity functions with respect to other parameters is similar. For example we derive the first- and second-order sensitivities $S_{G_{1}}^{T}$ and $S_{G_{1} G_{2}}^{T}$ :

$$
\begin{gathered}
S_{G_{1}}^{T}=-\frac{s^{2} C_{1} C_{2}+s\left[C_{1} G_{2}+C_{1} G_{2}(1-\mu)\right]}{s^{2} C_{1} C_{2}+s\left[(1-\mu) C_{1} G_{2}+C_{2}\left(G_{1}+G_{2}\right)\right]+G_{1} G_{2}}, \\
S_{G_{1} G_{2}}^{T}=\left(G _ { 1 } G _ { 2 } \left[\Delta_{(1+0)(1+4)} \Delta_{(1+4)(4+3),(1+0)(1+0)}\right.\right. \\
\times \Delta_{(4+3)(2+0),(1+0)(1+0)}+\Delta_{(1+0)(4+3)} \\
\left.\left.\quad \times \Delta_{(4+3)(1+4),(1+0)(1+0)} \Delta_{(1+4)(2+0),(1+0)(1+0)}\right]\right) \\
\times\left(\Delta^{2} \Delta_{(1+0)(2+0)}\right)^{-1},
\end{gathered}
$$

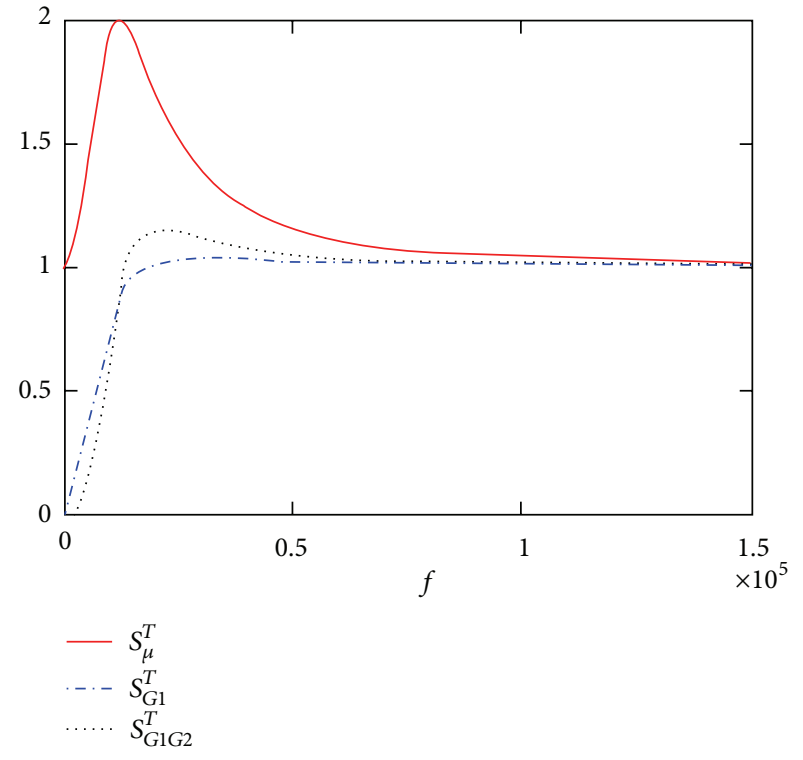

FIGURE 5: First- and second-order symbolic sensitivities versus frequency $f$.

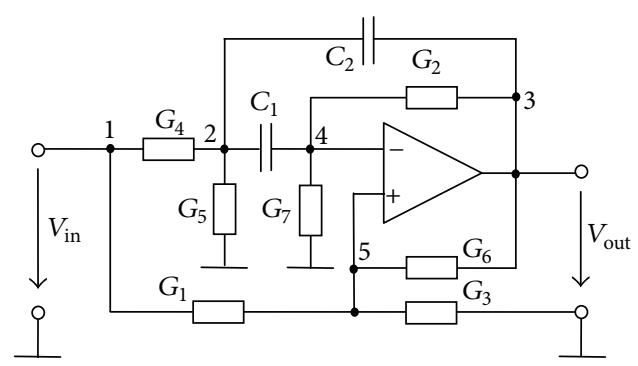

FIGURE 6: The STAR network.

where

$$
\begin{gathered}
\Delta_{(1+0)(2+0)}=\mu G_{1} G_{2}, \\
\Delta=s C_{2}\left(s C_{1}+G_{2}(1-\mu)\right)+s C_{1}\left(G_{2}+G_{1}\right)+G_{1} G_{2}, \\
\Delta_{(1+0)(1+4)}=s_{2}\left(C_{1} C_{2}\right)+s\left(C_{1} G_{2}(1-\mu)+C_{2} G_{2}\right) \\
\Delta_{(1+4)(4+3),(1+0)(1+0)}=-s C_{2}, \\
\Delta_{(4+3)(2+0),(1+0)(1+0)}=s\left(-\mu C_{1}\right)-\mu G_{1}, \\
\Delta_{(1+0)(4+3)}=s C_{2} G_{1}, \\
\Delta_{(4+3)(1+4),(1+0)(1+0)}=s\left(C_{1} \mu-C_{2}\right) \\
\Delta_{(1+4)(2+0),(1+0)(1+0)}=-\mu G_{2} 0 .
\end{gathered}
$$

Using Mathcad software symbolic sensitivity simulation results versus frequency $f$ are shown in Figure 5.

As it is seen, the symbolic sensitivity analysis technique based on the concept of high order summative cofactors and GPEM rules can be implemented in circuit design process and for circuit characterization, successfully. 

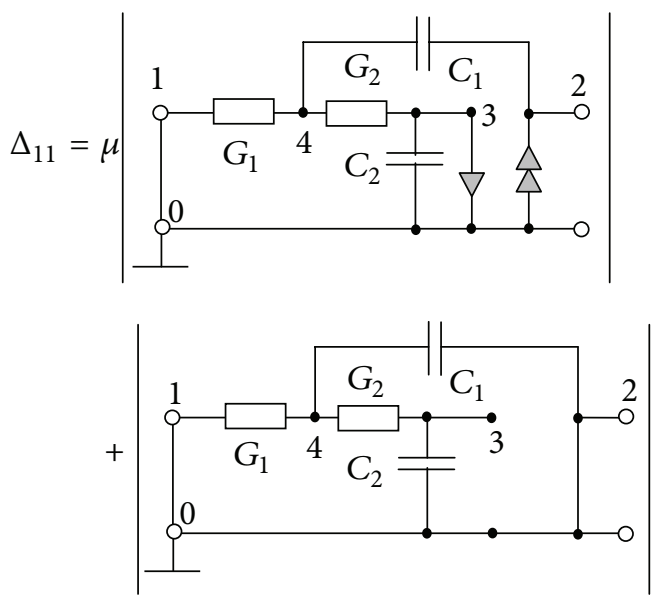

FIGURE 7

\section{Comparison Test}

4.1. The Comparison Conditions. In this section we present the comparison of sensitivity expressions obtained by means of three different approaches: the two-port transimpedance method [6], the method based on the modified Coates flowgraph [7], and the proposed technique. The test circuit is shown in Figure 6 [7].

Let us suppose that voltage transfer function $T(s)$ and the first- and second-order symbolic sensitivities with respect to parameters $G_{4}$ and $C_{2}$ are under consideration. We compare the formulae by following criteria in order to estimate the methods calculation efficiency: the number of arithmetical operations and occurrence of cancellations.

4.2. Symbolic Expressions Obtained by means of the GPEMBased Technique. The symbolic expressions $T(s), S_{G_{4}}^{T}, S_{s_{2}}^{T}$, and $S_{G_{4} s C_{2}}^{T}$ shown below are calculated by means of the symbolic circuit analysis program Cirsym. The sensitivity functions are presented here in two forms: Hoang form (10) and Bode form (Table 1).

The transfer function

$$
T(s)=\frac{\Delta_{(1+0)(3+0)}}{\Delta},
$$

where

$$
\begin{gathered}
y 1=s * C 1 ; \quad y 2=s * C 2 ; \\
\Delta_{(1+0)(3+0)}=y 1 *((G 7+G 2+G 5+y 2) *(G 1) \\
\quad-G 4 *(G 6+G 3)) \\
+(G 7+G 2) * G 1 *(G 5+y 2+G 4) ; \\
\Delta=y 1 \\
*((G 7+G 5+G 4) *(-G 6)+(G 2+y 2) *(G 3+G 1)) \\
+((G 5+G 4+y 2)) *((G 3+G 1) *(G 2)-G 6 * G 7) .
\end{gathered}
$$

The sensitivity functions in Hoang form

$$
S_{G_{4}}^{T}=G_{4} \cdot\left(\frac{\Delta_{(1+0)(3+0)}^{\infty}}{\Delta_{(1+0)(3+0)}}-\frac{\Delta^{\infty}}{\Delta}\right),
$$

where

$$
\begin{gathered}
y 1=s * C 1 ; \quad y 2=s * C 2 ; \\
\Delta_{(1+0)(3+0)}^{\infty}=y 1 *(-(G 3+G 6))+G 1 *(G 7+G 2) ; \\
\Delta^{\infty}=(G 3+G 1) *(G 2)-G 6 *(G 7+y 1) . \\
S_{s C_{2}}^{T}=s C_{2} \cdot\left(\frac{\Delta_{(1+0)(3+0)}^{\infty}}{\Delta_{(1+0)(3+0)}}-\frac{\Delta^{\infty}}{\Delta}\right),
\end{gathered}
$$

where

$$
\begin{gathered}
y 1=s * C 1 ; \quad y 2=s * C 2 ; \\
\Delta_{(1+0)(3+0)}^{\infty}=G 1 *(G 7+G 2+y 1) ; \\
\Delta^{\infty}=(G 2+y 1) *((G 3+G 1))-G 6 * G 7 \\
S_{G_{4} s C_{2}}^{T}=G 4 * y 2 \\
*\left(\Delta_{(1+0)(3+0)}^{\infty} * \Delta * \Delta-\Delta^{s C_{2} \rightarrow \infty} * \Delta_{(1+0)(3+0)}^{G_{4} \rightarrow \infty}\right. \\
* \Delta-\Delta_{(1+0)(3+0)}^{s C_{2} \rightarrow \infty} * \Delta^{G_{4} \rightarrow \infty} \\
* \Delta-\Delta{ }_{(1+0)(3+0)} * \Delta^{\infty} * \Delta+2 \\
\left.* \Delta_{(1+0)(3+0)} * \Delta^{G_{4} \rightarrow \infty} * \Delta^{s C_{2} \rightarrow \infty}\right) \\
\left.\times\left(\Delta_{(1+0)(3+0)} * \Delta * \Delta\right)^{-1}\right),
\end{gathered}
$$

where

$$
\begin{gathered}
y 1=s * C 1 ; \quad y 2=s * C 2 ; \\
\Delta_{(1+0)(3+0)}^{G_{4} \rightarrow \infty}=y 1 *(-(G 6+G 3))+G 1 *(G 7+G 2) ; \\
\Delta_{(1+0)(3+0)}^{s C_{2} \rightarrow \infty}=G 1 *(G 7+G 2+y 1) ; \\
\Delta_{(1+0)(3+0)}^{\infty}=0 ; \\
\Delta^{G_{4} \rightarrow \infty}=(G 3+G 1) *(G 2)-G 6 *(G 7+y 1) ; \\
\Delta^{s C_{2} \rightarrow \infty}=(G 2+y 1) *((G 3+G 1))-G 6 * G 7 ; \\
\Delta^{\infty}=0 .
\end{gathered}
$$

The sensitivity functions in Bode form

$$
S_{G_{4}}^{T}=\frac{-G_{4} \Delta_{(1+0)(1+2)} \Delta_{(1+2)(3+0),(1+0)(1+0)}}{\Delta \Delta_{(1+0)(3+0)}}
$$




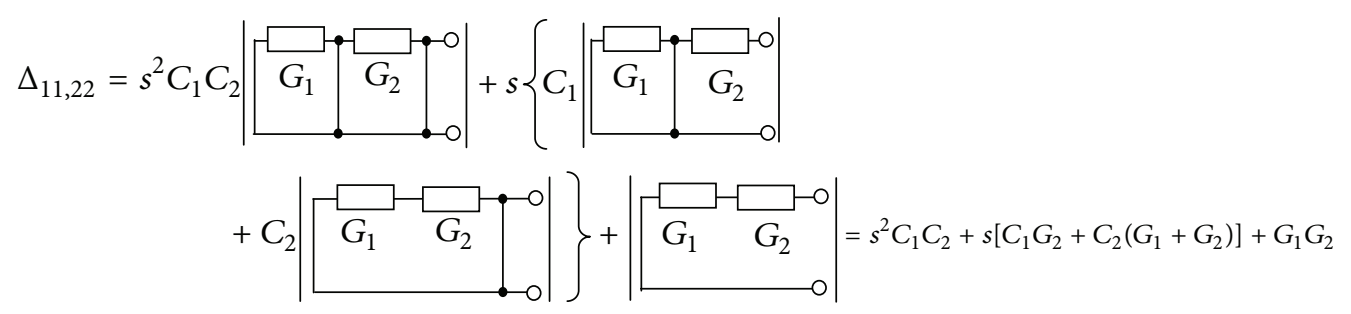

Figure 8

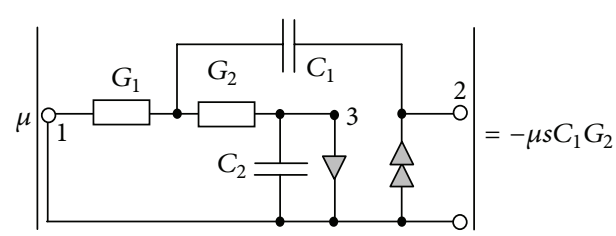

Figure 9

where

$$
\begin{aligned}
& y 1=s * C 1 ; \quad y 2=s * C 2 ; \\
& \Delta_{(1+0)(1+2)}=G 1 *(y 2 *(-G 7)+G 2 * G 5) \\
& +y 1 *((G 2+y 2) *(G 3)-G 6 *(G 7+G 5)) \\
& +((G 5+y 2)) *(G 2 *(G 3)-G 6 * G 7) ; \\
& \Delta_{(1+2)(3+0),(1+0)(1+0)}=y 1 *(G 6+G 3+G 1), \\
& S_{s C_{2}}^{K}=\frac{-y_{2} \Delta_{(1+0)(2+3)} \Delta_{(2+3)(3+0),(1+0)(1+0)}}{\Delta \Delta_{(1+0)(3+0)}}
\end{aligned}
$$

where

$$
\begin{gathered}
y 1=s * C 1 ; \quad y 2=s * C 2 ; \\
\Delta_{(1+0)(2+3)=} y 1 *((G 7+G 5) *(-G 1)+G 3 * G 4) \\
+G 4 *(G 1 *(-G 7)+G 2 *(G 3)-G 6 * G 7) \\
-G 1 * G 5 *(G 7+G 2) ; \\
\Delta_{(2+3)(3+0),(1+0)(1+0)}=(-y 1 *(G 6+G 3+G 1)) \\
S_{G_{4} s C_{2}}^{T}=\left(G _ { 4 } y _ { 2 } \left[\Delta_{(1+0)(1+2)} \Delta_{(1+2)(2+3),(1+0)(1+0)}\right.\right. \\
\quad \times \Delta_{(2+3)(3+0),(1+0)(1+0)}+\Delta_{(1+0)(2+3)} \\
\left.\left.\times \Delta_{(2+3)(1+2),(1+0)(1+0)} \Delta_{(1+2)(3+0),(1+0)(1+0)}\right]\right) \\
\times\left(\Delta^{2} \Delta_{(1+0)(2+0)}\right)^{-1},
\end{gathered}
$$

where

$$
\begin{aligned}
& y 1=s * C 1 ; \quad y 2=s * C 2 ; \\
& \Delta_{(1+0)(1+2)}=G 1 *(y 2 *(-G 7)+G 2 * G 5) \\
& +y 1 *((G 2+y 2) *(G 3)-G 6 *(G 7+G 5)) \\
& +((G 5+y 2)) *(G 2 *(G 3)-G 6 * G 7) \text {; } \\
& \Delta_{(1+2)(2+3),(1+0)(1+0)}=y 1 *(-(G 3+G 1)) \\
& -((G 3+G 1) *(G 2)-G 6 * G 7) ; \\
& \Delta_{(2+3)(3+0),(1+0)(1+0)}=-y 1 *(G 6+G 3+G 1) ; \\
& \Delta_{(1+0)(2+3)}=y 1 *((G 7+G 5) *(-G 1)+G 3 * G 4) \\
& +G 4 *(G 1 *(-G 7)+G 2 *(G 3)-G 6 * G 7) \\
& -G 1 * G 5 *(G 7+G 2) \text {; } \\
& \Delta_{(2+3)(1+2),(1+0)(1+0)}=y 1 *(G 6) \\
& -((G 3+G 1) *(G 2)-G 6 * G 7) ; \\
& \Delta_{(1+2)(3+0),(1+0)(1+0)}=y 1 *(G 6+G 3+G 1) .
\end{aligned}
$$

4.3. Symbolic Expressions Obtained by means of Modified Coates Flow-Graph Concept. The Coates flow-graph is useful and often used in the network theory. The modification of Coates flow-graph provides the symbolic sensitivity analysis of nullor-based equivalent circuits [7]. We will show here that modification of Coates flow-graph is corresponding to implementation of the nullor extraction formula (8).

Let us consider the flow-graph presented in Figure 8(a) (see publication [7]) for the passive part of the equivalent circuit of STAR network shown in Figure 6. The oriented norator connected between nodes 3 and 0 . The oriented nullator connected between nodes 5 and 4 .

First, we choose the node 3 as supporting and make the norator short-circuited:

(1) the ends of all incoming edges $\left(-s C_{2}\right.$ and $\left.-G_{2}\right)$ into node 3 are moved to node 0 ;

(2) the self-loop Y33 transforms into the incoming edge into node 0 ;

(3) the edges connected to node 0 are not shown on graph. 
Second, we choose the node 5 as supporting and make the nullator short-circuited:

(1) the end of incoming edge $-G_{6}$ into node 5 is moved to node 4;

(2) the self-loop Y55 transformed into the incoming edge into node 4.

Third, we combine the two supporting nodes 3 and 5:

(1) the edge $-G_{6}$ between nodes 3 and 5 transforms into the self-loop in the new node named by 3 ;

(2) the parallel codirectional edges $-G_{6}$ and $Y 55$ are combined into the edge $Y 55$.

Consequently we obtained the modified Coates flowgraph shown in Figure 8(b) (see publication [7]). Note that in case of single supporting node the procedure of nodes combining is not needed.

The estimation process of the determinant sign of modified Coates flow-graph is not formalized in [7]. It leads to complications of method usage and high error probability.

Implementation of the oriented nullor concept [16] provides the simple rule for estimation of the sign of Coates graph determinant: the sign is positive (negative), if norator and nullator have the same (different) orientations against the supporting nodes. In case of two supporting nodes the inverted rule is needed.

The symbolic formulae $T(s), S_{\mathrm{G}_{4}}^{T}, S_{s C_{2}}^{T}$, and $S_{\mathrm{G}_{4} s C_{2}}^{T}$ presented here were replicated from [7] with correction of typing error in subexpression $Y 55$ and sign of determinants:

$$
\begin{gathered}
T(s)=(-Y 22 * G 1 * Y 44+y 1 * y 1 \\
* G 1+y 1 * Y 55 * G 4)
\end{gathered}
$$

$$
\begin{aligned}
& \times(Y 22 * G 6 * Y 44-Y 22 * G 2 * Y 55-y 1 \\
& \quad * y 1 * G 6-y 1 * y 2 * Y 55)^{-1}
\end{aligned}
$$

where

$$
\begin{gathered}
Y 22=(G 4+G 5+y 1+y 2) ; \\
Y 44=(G 2+G 7+y 1) ; \\
Y 55=(G 1+G 3+G 6) ; \\
y 1=s * C 1 ; \quad y_{2}=s * C 2 .
\end{gathered}
$$

Let us substitute the subexpressions $Y 22, Y 44$, and $Y 55$ into the transfer function (36):

$$
\begin{aligned}
T(s)=(( & (G 4+G 5+y 1+y 2) * G 1 \\
& *(G 2+G 7+y 1)+y 1 * y 1 * G 1 \\
& +y 1 *(G 1+G 3+G 6) * G 4)) \\
\times & (((G 4+G 5+y 1+y 2) * G 6 \\
& *(G 2+G 7+y 1)-(G 4+G 5+y 1+y 2) \\
& * G 2 *(G 1+G 3+G 6)-y 1 * y 1 * G 6 \\
& -y 1 * y 2 *(G 1+G 3+G 6)))^{-1} .
\end{aligned}
$$

It is easy to verify that the expanded expressions of the numerator and denominator will consist of the 16 and 28 summands correspondingly. After simplification only 12 terms in numerator and 16 terms in denominator will remain. So, the transfer function (36) includes the 8 pairs of cancellation sum-of-product terms:

$$
\begin{aligned}
S_{G_{4}}^{T}= & \frac{G 4}{(-Y 22 * G 1 * Y 44+y 1 * y 1 * G 1+y 1 * Y 55 * G 4)} \\
& *\left(1-\frac{(Y 44 *(G 4 * G 6-G 1 * y 2)-G 2 * G 4 * Y 55-G 2 * G 1 * y 1)}{(Y 22 * G 6 * Y 44-Y 22 * G 2 * Y 55-Y 1 * y 1 * G 6-y 1 * y 2 * Y 55)}\right) * y 1 * Y 55, \\
S_{s C_{2}}^{T}= & \left(\begin{array}{l}
(-Y 22 * G 1 * Y 44+y 1 * y 1 * G 1+y 1 * Y 55 * G 4) \\
\end{array}\right. \\
& \left.\times(Y 22 * G 6 * Y 44-Y 22 * G 2 * Y 55-y 1 * y 1 * G 6-y 1 * y 2 * Y 55)^{-1}\right) \\
& *(-Y 44 *(G 4 * G 6-G 1 * y 2)+G 2 * G 4 * Y 55+G 2 * G 1 * y 1-Y 22 * G 1 * Y 44+y 1 * y 1 \\
& * G 1+y 1 * Y 55 * G 4) * y 1 * Y 55,
\end{aligned}
$$




$$
\begin{aligned}
S_{G_{4} s C_{2}}^{T}=( & \left.\frac{-G 4 * y 2}{(}-Y 22 * G 1 * Y 44+y 1 * y 1 * G 1+y 1 * Y 55 * G 4\right) \\
& \left.\times\left(Y 22 * G 6 * Y 44-Y 22 * G 2 * Y 55-y 1^{2} * G 6-y 1 * y 2 * Y 55\right)^{-1}\right) \\
* & (-(-Y 44 * G 6+Y 55 * G 2) * y 1 * Y 55 \\
& * \frac{(-Y 44 *(G 4 * G 6-G 1 * y 2)+G 2 * G 4 * Y 55+G 2 * G 1 * y 1-Y 22 * G 1 * Y 44+y 1 * y 1 * G 1+y 1 * Y 55 * G 4)}{(Y 22 * G 6 * Y 44-Y 22 * G 2 * Y 55-y 1 * y 1 * G 6-y 1 * y 2 * Y 55)} \\
& -(-Y 44 * G 6+Y 55 * G 2+y 1 * Y 55) * y 1 * Y 55 \\
& \left.*\left(1-\frac{(Y 44 *(G 4 * G 6-G 1 * y 2)-G 2 * G 4 * Y 55-G 2 * G 1 * y 1)}{(Y 22 * G 6 * Y 44-Y 22 * G 2 * Y 55-y 1 * y 1 * G 6-y 1 * y 2 * Y 55)}\right)\right) .
\end{aligned}
$$

4.4. Symbolic Expressions Obtained by means of TwoPort Transimpedance Method. The sequences of expressions, derived by symbolic analysis program STAINS [6], are listed below. The calculation of second-order sensitivity is not support in the current version of the program. So we present here only the transfer function and two first-order symbolic sensitivities with respect to parameters $G_{4}$ and $C_{2}$ :

$$
T(s)=\frac{Z o i}{Z i i},
$$

where

$$
\begin{gathered}
y 1=s * C 1 ; \quad y 2=s * C 2 ; \\
x 1=-G 1 ; \quad x 2=-G 2-G 6 ; \\
x 3=G 1+G 3+G 6 ; \quad d 1=\frac{G 2}{(y 1)} ; \\
x 4=G 4 * d 1 ; \\
x 5=G 2+G 6+y 2+y 2 * d 1 ; \\
x 6=-y 2-(G 4+G 5+y 1+y 2) * d 1 ; \\
d 7=x 1+G 4 * d 2 ; \quad x 8=x 2+y 2 * d 2 ; \\
x 9=-y 1-(G 4+G 5+y 1+y 2) * d 2 ; \\
d 3=-\frac{G 1}{(x 3)} ; \quad x 10=G 1+G 4-x 7 * d 3 ; \\
x 11=-x 8 * d 3 ; \quad x 12=-G 4-x 9 * d 3 ; \\
d 4=-\frac{G 6}{(x 3)} ; \quad x 13=x 4-x 7 * d 4 ; \\
x 14=x 5-x 8 * d 4 ; \quad x 15=x 6-x 9 * d 4 ;
\end{gathered}
$$

$$
\begin{array}{lr}
Z 21=1 * x 12 ; & Z o i=Z 21 \\
Z 11=-1 * x 15 ; & Z i i=Z 11 .
\end{array}
$$

After substitution of the subexpressions into (42) we obtain the transfer function expression as follows:

$$
\begin{aligned}
& T(s)=(-1 *(G 4 *(G 1+G 3+G 6)-G 1 \\
& *((-s * C 1)-(G 4+G 5+s *(C 1+C 2)) \\
& \left.\left.\left.*\left(-\frac{(G 2+G 7+s * C 1)}{(s * C 1)}\right)\right)\right)\right) \\
& \times(-1 *(G 6 *((-s * C 1) \\
& -(G 4+G 5+s *(C 1+C 2)) \\
& \left.*\left(-\frac{(G 2+G 7+s * C 1)}{(s * C 1)}\right)\right) \\
& +(-s * C 2-(G 4+G 5+s *(C 1+C 2)) \\
& \left.*\left(\frac{G 2}{(s * C 1)}\right)\right) \\
& *(G 1+G 3+G 6)))^{-1} \text {. }
\end{aligned}
$$

The expansion of the transfer function numerator and denominator leads to obtaining the expressions with 16 and 28 terms correspondingly. Just as formula (36) the transfer 
function (42) includes the 8 pairs of cancellation sum-ofproduct terms:

$$
S_{G_{4}}^{T}=G 4 * Z k i * \frac{(Z o k * Z i i-Z i k * Z o i)}{(Z o i * D 00 * Z i i)},
$$

where

$$
\begin{aligned}
& y 1=s * C 1 ; \quad y 2=s * C 2 ; \\
& x 1=-G 1 ; \quad x 2=-G 2-G 6 ; \\
& x 3=G 1+G 3+G 6 ; \quad d 1=-\frac{G 1}{(x 3)} \\
& x 4=G 1+G 4-x 1 * d 1 ; \quad x 5=-x 2 * d 1 ; \\
& x 6=y 1 * d 1-G 4 ; \quad x 7=-(G 2+G 7+y 1) * d 1 ; \\
& d 2=-\frac{G 6}{(x 3)} ; \quad x 8=-x 1 * d 2 ; \\
& x 9=G 2+G 6+y 2-x 2 * d 2 \\
& x 10=y 1 * d 2-y 2 \\
& x 11=-G 2-(G 2+G 7+y 1) * d 2 ; \\
& Z 21=1 *(x 6 * y 1+x 7 *(G 4+G 5+y 1+y 2)) ; \\
& Z o i=Z 21 ; \\
& Z 11=-1 *(x 10 * y 1+x 11 *(G 4+G 5+y 1+y 2)) \\
& \text { Zii }=\text { Z11; } \quad Z 13=1 *(x 8 * y 1-x 11 * G 4) ; \\
& Z i k=Z 11-Z 13 ; \quad Z 23=-1 *(x 4 * y 1-x 7 * G 4) ; \\
& Z o k=Z 21-Z 23 ; \quad Z 31=1 *(x 6 * x 11-x 7 * x 10) \text {; } \\
& Z k i=Z 11-Z 31 \\
& D 00=x 4 * Z 11+x 8 * Z 21-G 4 * Z 31 . \\
& S_{s C_{2}}^{T}=y 2 * Z k i * \frac{(Z i k * Z o i-Z o k * Z i i)}{(Z o i * D 00 * Z i i)},
\end{aligned}
$$

where

$$
\begin{gathered}
y 1=s * C 1 ; \quad y 2=s * C 2 ; \\
x 1=-G 1 ; \quad x 2=-G 2-G 6 ; \\
x 3=G 1+G 3+G 6 ; \quad d 1=-\frac{G 1}{(x 3)} \\
x 4=G 1+G 4-x 1 * d 1 ; \quad x 5=-x 2 * d 1 ; \\
x 6=y 1 * d 1-G 4 ; \quad x 7=-(G 2+G 7+y 1) * d 1 ; \\
d 2=-\frac{G 6}{(x 3)} ; \quad x 8=-x 1 * d 2 ;
\end{gathered}
$$

TABLE 3: Comparison of symbolic analysis methods efficiency.

\begin{tabular}{lccc}
\hline Symbolic function & Symbolic analysis method & $\mathrm{M} / \mathrm{D}$ & $\mathrm{A} / \mathrm{S}$ \\
\hline \multirow{4}{*}{$T(s)$} & GPEM (22) & 14 & 20 \\
& MCFG (36) & 17 & 13 \\
& TI (42) & 29 & 34 \\
\hline \multirow{4}{*}{$S_{G_{4}}^{T}$} & GPEM (Hoang form) (24) & 20 & 28 \\
& GPEM (Bode form) (30) & 27 & 32 \\
& MCFG (39) & 28 & 17 \\
$S_{s C_{2}}^{T}$ & TI (45) & 37 & 42 \\
\hline & GPEM (Hoang form) (26) & 19 & 26 \\
& GPEM (Bode form) (32) & 27 & 33 \\
& MCFG (40) & 34 & 20 \\
$S_{G_{4} s C_{2}}^{T}$ & TI (47) & 37 & 43 \\
& GPEM (Hoang form) (28) & 50 & 72 \\
& GPEM (Bode form) (34) & 54 & 63 \\
& MCFG (41) & 68 & 38 \\
\hline
\end{tabular}

$$
\begin{gathered}
x 9=G 2+G 6+y 2-x 2 * d 2 ; \\
x 10=y 1 * d 2-y 2 ; \\
x 11=-G 2-(G 2+G 7+y 1) * d 2 ; \\
Z 21=1 *(x 6 * y 1+x 7 *(G 4+G 5+y 1+y 2)) ; \\
Z 11=-1 *(x 10 * y 1+x 11 *(G 4+G 5+y 1+y 2)) ; \quad Z 12=-0 ; \\
Z i i=Z 11 ; \\
Z 13=1 *(x 8 * y 1-x 11 * G 4) ; \\
Z i k=Z 12-Z 13 ; \quad Z 22=0 ; \\
Z 23=-1 *(x 4 * y 1-x 7 * G 4) \\
Z o k=Z 22-Z 23 ; \\
Z 31=1 *(x 6 * x 11-x 7 * x 10) ; \\
Z k i=Z 21-Z 31 ; \\
D 00=x 4 * Z 11+x 8 * Z 21-G 4 * Z 31
\end{gathered}
$$

4.5. Efficiency Comparison. In Table 3 we present the comparison of symbolic analysis methods efficiency by the number of arithmetical operations. The multiplication and division are denoted as M/D and the addition and subtraction are denoted as A/S. The method based on modified Coates flow-graph is denoted as MCFG and the transimpedance method is denoted as TI.

As can be seen, the symbolic expressions obtained by transimpedance method consist of the largest arithmetical operations amount. The results of MCFG-computation are comparable to expressions presented in Bode form obtained by GPEM. The symbolic sensitivity functions presented in 
Hoang form are superior by number of arithmetical operations.

Both Bode and Hoang forms implement the four minor calculations. But the presentation of sensitivity function in Hoang form provides the optimal compact size of symbolic expressions. On the other hand the formulae in Bode form for special topological cases proposed in this paper provide the significant simplification of circuit analysis in many cases.

\section{Conclusions}

In this paper we have presented a method of computation the circuit functions sensitivities in symbolic form. The method requires neither matrix nor ordinary graph description of the circuit. The main advantages of the proposed technique are that it is cancellation-free and provided the compact size of obtaining sensitivity expression as distinct from other methods like the two-port transimpedance method and the method based on the modified Coates flow-graph. It is shown that presentation of sensitivity function in Hoang form provides the optimal compact size of symbolic expressions, and the formulae in Bode form provide the significant simplification of active circuit symbolic analysis. The process of calculation of first- and second-order and multiparameter symbolic sensitivity is automated by computer program Cirsym and allows obtaining all symbolic sensitivities simultaneously.

\section{Conflict of Interests}

The authors declare that there is no conflict of interests regarding the publication of this paper.

\section{References}

[1] H. W. Bode, Network Analysis and Feedback Amplifier Design, D. Van Nostrand, New York, NY, USA, 1945.

[2] S. R. Parker, E. Peskin, and P. Chirlian, "Application of a bilinear theorem to network sensitivity," IEEE Transactions on Circuit Theory, vol. 12, no. 3, pp. 448-450, 1965.

[3] E. V. Sorensen, "General relations governing the exact sensitivity of linear networks," Proceedings of the Institution of Electrical Engineers, vol. 114, no. 9, pp. 1209-1212, 1967.

[4] S.-D. Shieu and S.-P. Chan, "Topological formulation of symbolic network functions and sensitivity analysis of active networks," IEEE Transactions on Circuits and Systems, vol. 21, no. 1, pp. 39-45, 1974.

[5] S. Hoang, "Direct topological method in network sensitivity analysis," Archiwum Elektrotechniki, vol. 21, no. 4, pp. 767-784, 1975.

[6] F. Balik and B. Rodanski, "Calculation of symbolic sensitivities for large-scale circuits in the sequence of expressions form via the transimpedance method," Analog Integrated Circuits and Signal Processing, vol. 40, no. 3, pp. 265-276, 2004.

[7] I. Asenova and F. Balik, "Multiparameter symbolic sensitivity analysis of active circuits by using nullor model and modified Coates flow graph," in Proceedings of the 9th International Conference on ELEKTRO, pp. 401-406, Rajeck Teplice, Slovakia, May 2012.
[8] H. Yang, M. Ranjan, W. Verhaegen, M. Ding, R. Vemuri, and G. Gielen, "Efficient symbolic sensitivity analysis of analog circuits using element coefficient diagrams," in Proceedings of the Asia South-Pacific Design Automation Conference (ASPDAC '05), pp. 230-235, Yokohama, Japan, 2005.

[9] G. Shi and X. Meng, "Variational analog integrated circuit design via symbolic sensitivity analysis," in Proceedings of the IEEE International Symposium on Circuits and Systems (ISCAS '09), pp. 3002-3005, Taipei, Taiwan, May 2009.

[10] S. Rodriguez-Chavez, A. A. Palma-Rodriguez, E. Tlelo-Cuautle, and S. X.-D. Tan, "Graph-based symbolic and symbolic sensitivity analysis of analog integrated circuits," in Analog/RF and Mixed-Signal Circuit Systematic Design, vol. 233 of Lecture Notes in Electrical Engineering, pp. 101-122, Springer, 2013.

[11] L. Mandache, M. Iordache, L. Dumitriu, I. Gabriela Sirbu, and D. Niculae, "Sensitivity analysis of analog circuits based on a modified nodal approach," in Proceedings of the International Conference on Optimization of Electrical and Electronic Equipment (OPTIM '14), pp. 83-88, Bran, Romania, May 2014.

[12] G. E. Alderson and P. M. Lin, "Computer generation of symbolic network functions-a new theory and implementation," IEEE Transactions on Circuit Theory, vol. 20, no. 1, pp. 48-56, 1973.

[13] P. M. Lin, Symbolic Network Analysis, Elsevier, Amsterdam, The Netherlands, 1991.

[14] S. Lasota, "Parameter decision diagrams in the analysis and structural synthesis: part I, III," in Proceedings of the 10th International Workshop on Symbolic and Numerical Methods, Modeling and Application to Circuit Design (SMACD '08), pp. 149-157, 180-187, Erfurt, Germany, 2008.

[15] S. Lasota, "Multilevel hierarchical always cancellation-free symbolic analysis method for large electric networks," Elektronika: Konstrukcje, Technologie, Zastosowania, vol. 54, no. 2, pp. 51-57, 2013.

[16] V. V. Filaretov and A. S. Korotkov, "Generalized parameter extraction method in symbolic network analysis," in Proceedings of the European Conference on Circuits Theory and Design (ECCTD '03), vol. 2, pp. 406-409, Kraków, Poland, September 2003.

[17] V. V. Filaretov and A. S. Korotkov, "Generalized parameter extraction method in case of multiple excitation," in Proceedings of the 8th International Workshop on Symbolic Methods and Applications to Circuit Design (SMACD '04), pp. 8-11, Wrocław, Poland, September 2004.

[18] V. Filaretov and K. Gorshkov, "The generalization of the extra element theorem for symbolic circuit tolerance analysis," Journal of Electrical and Computer Engineering, vol. 2011, Article ID 652706, 5 pages, 2011.

[19] V. Filaretov and K. Gorshkov, "Transconductance realization of block-diagrams of electronic networks," in Proceedings of the International Conference on Signals and Electronic Systems (ICSES '08), pp. 261-264, Krakow, Poland, September 2008.

[20] V. Filaretov, K. Gorshkov, and A. Mikheenko, "A circuit synthesis technique based on network determinant expansion," in Proceedings of the International Conference on Synthesis, Modeling, Analysis and Simulation Methods and Applications to Circuit Design (SMACD '12), pp. 293-296, Seville Sevilla, Spain, September 2012.

[21] V. V. Filaretov and K. S. Gorshkov, "Topological analysis of active networks containing pathological mirror elements," in Proceedings of the IEEE 33rd International Scientific Conference Electronics and Nanotechnology (ELNANO '13), pp. 293-296, Kiev, Ukrain, April 2013. 
[22] R. Dmytryshyn and A. Kubaszek, "Sequence of expressions generation for the repetitive analysis acceleration," in Proceedings of the International Workshop on Symbolic Methods, Modeling and Application in Circuit Design (SMACD '98), pp. 154-159, Kaiserslautern, Germany, 1998.

[23] J. A. Starzyk and A. Konczykowska, "Flowgraph analysis of large electronic networks," IEEE Transactions on Circuits and Systems, vol. 33, no. 3, pp. 302-315, 1986.

[24] B. Rodanski, "Computational efficiency of symbolic sequential formulae," in Proceedings of the 6th International Workshop on Symbolic Methods and Applications to Circuit Design (SMACD '00), pp. 45-50, Lisbon, Portugal, October 2000.

[25] J. Vlach and K. Singhal, Computer Methods for Circuit Analysis and Design, Van Nostrand Reinhold Company, New York, NY, USA, 1983. 

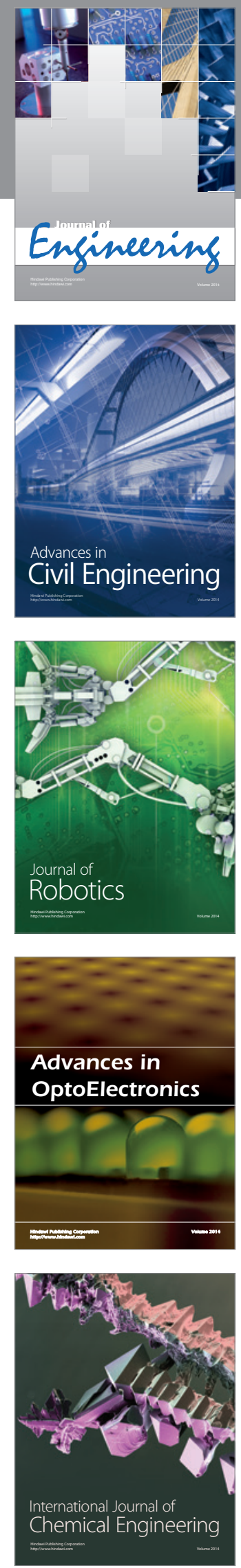

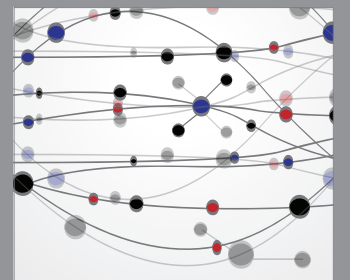

The Scientific World Journal
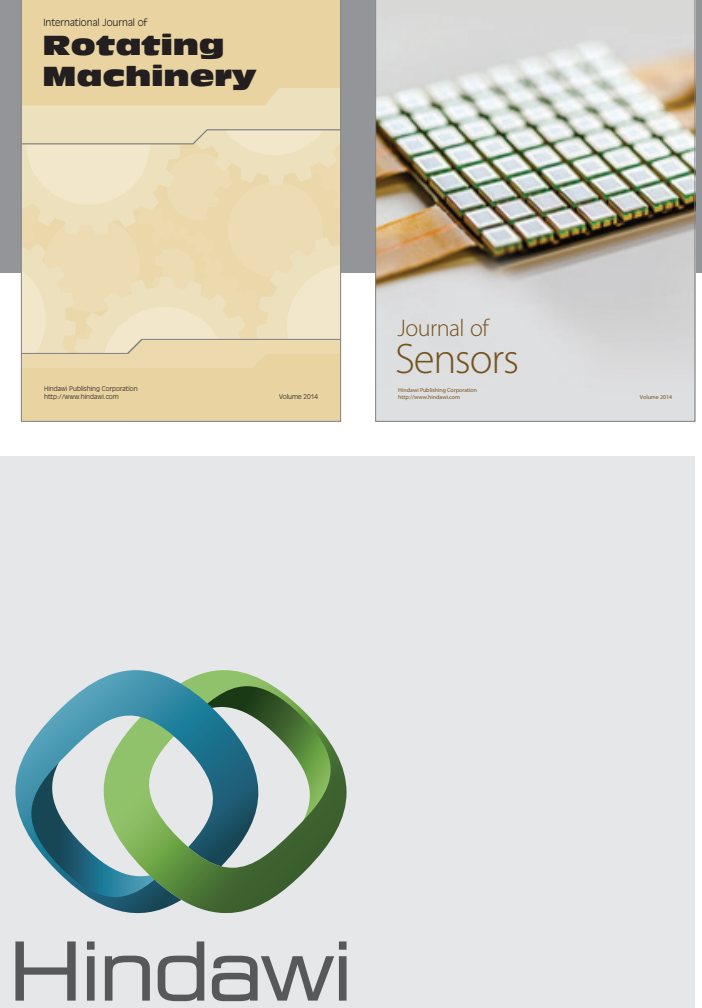

Submit your manuscripts at http://www.hindawi.com
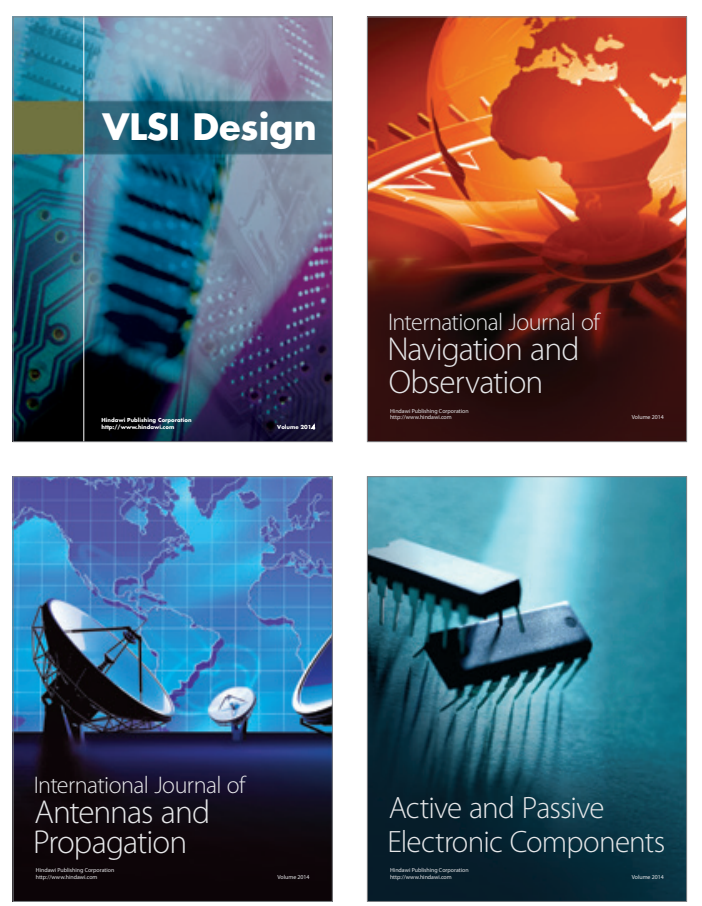
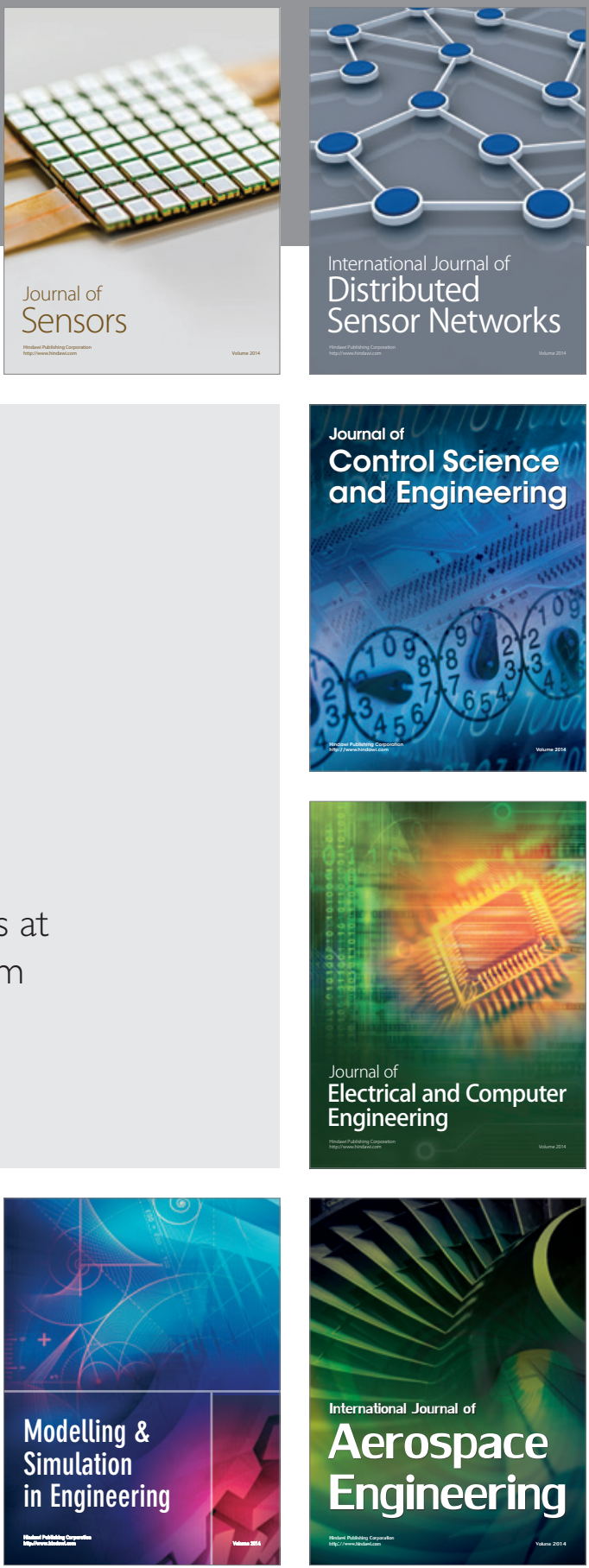

Journal of

Control Science

and Engineering
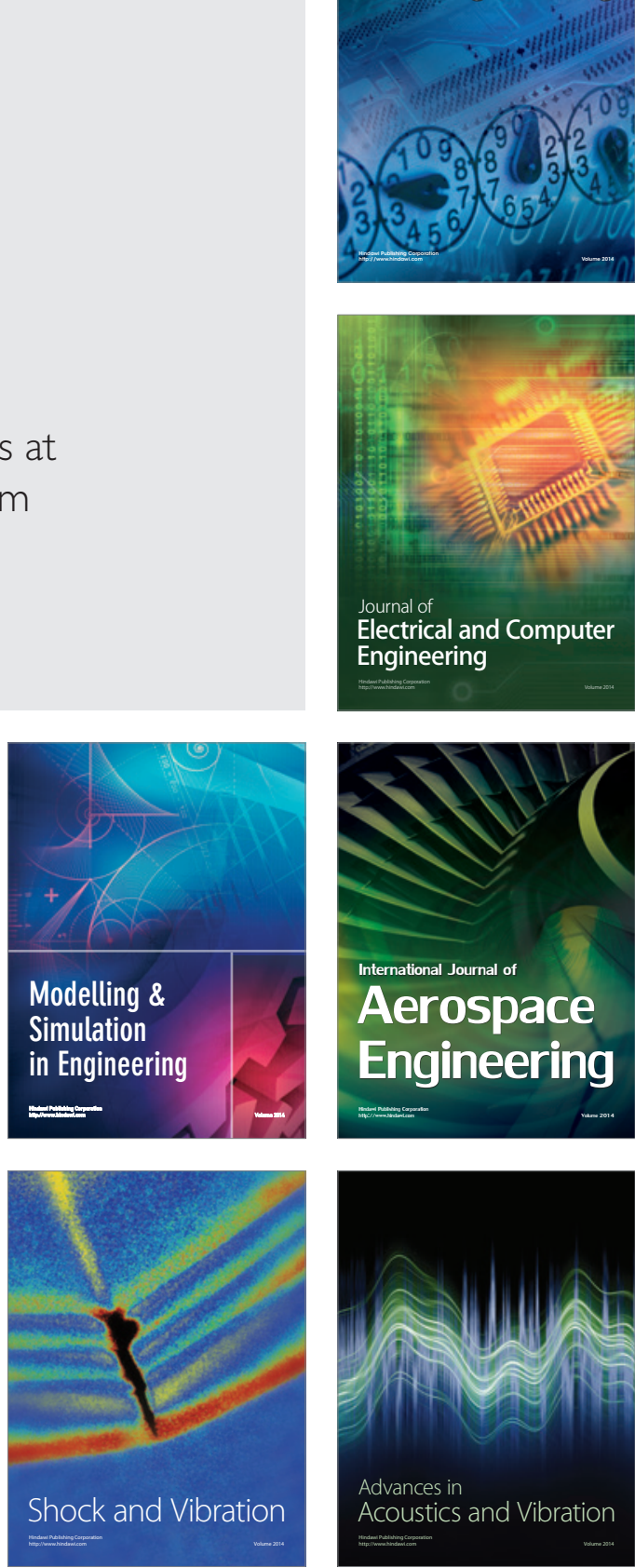